Henssler/Willemsen/Kalb (Hrsg.) · Arbeitsrecht Kommentar 



\section{Arbeitsrecht Kommentar}

herausgegeben von

Prof. Dr. Martin Henssler

Universität zu Köln

Prof. Dr. Heinz Josef Willemsen Rechtsanwalt, Fachanwalt für Arbeitsrecht, Düsseldorf

Dr. Heinz-Jürgen Kalb

Vizepräsident des LAG Köln a.D.

7. Auflage

2016

\section{ottoschmidt}





\section{Bearbeiter der 7. Auflage}

Prof. Dr. Georg Annuß, LL.M., RA, München

Prof. Klaus Bepler, VorsRiBAG a.D., Berlin

Prof. Dr. Claudia Bittner, LL.M.,

Ri'inLSG, Darmstadt

Dr. Susanne Clemenz,

RAin FAArbR, Gütersloh

Dr. Martin Diller, RA FAArbR, Stuttgart

Dr. Boris Dzida, RA FAArbR, Hamburg

Dr. Benno Alexander Fischer, LL.M.,

RA u. StB, Düsseldorf

Dr. Hans Jörg Gäntgen,

Vizepräs. LAG, Köln

Prof. Dr. Björn Gaul, RA FAArbR, Köln

Prof. Dr. Richard Giesen,

Universitätsprofessor, München

Dr. Michael Gotthardt,

VorsRiLAG, Düsseldorf

Prof. Dr. Martin Henssler,

Universitätsprofessor, Köln

Dr. Carmen Silvia Hergenröder,

RAin, Daxweiler

Prof. Dr. Curt Wolfgang Hergenröder,

Universitätsprofessor,

Stv.Vors. KArbG, Mainz

Prof. Dr. Klaus-Stefan Hohenstatt,

RA FAArbR, Hamburg

Dr. Heinz-Jürgen Kalb,

Vizepräs. LAG Köln a.D.

Prof. Dr. Michael Kliemt,

RA FAArbR, Düsseldorf

Prof. Dr. Rüdiger Krause,

Universitätsprofessor, Göttingen

Prof. Dr. Mark Lembke, LL.M.,

RA FAArbR, Attorney-at-Law (New York), Frankfurt/M.

Dr. Josef Molkenbur, VorsRiLAG, Halle/S.

Dr. Thomas Müller-Bonanni, LL.M., RA, Düsseldorf
Bernhard Nimscholz,

RA, Ltd VDir a.D., Haan

Prof. Dr. Susanne Peters-Lange, Hochschule Bonn-Rhein-Sieg, Hennef

Martin Quecke,

VorsRiLAG, Düsseldorf

Prof. Dr. Hermann Reichold,

Universitätsprofessor, Tübingen

Dr. Maren Rennpferdt, Ri'inBAG, Erfurt

Prof. Dr. Oliver Ricken,

Universitätsprofessor, Bielefeld

Dr. Sebastian Roloff, RiArbG Köln

Dr. Hans-Jürgen Rupp, Richter, Ulm

Prof. Dr. Bernd Sandmann,

RA FAArbR, Augsburg

Reinhard Schinz, VorsRiLAG, Berlin

Dr. Johannes Schipp,

RA FAArbR, Gütersloh

Harald Schliemann,

Thüringer Justizminister a.D.

VorsRiBAG i.R., RA Isernhagen/Hannover

Dr. Werner Schmalenberg,

RA FAArbR u. Notar a.D., Bremen

Prof. Dr. Christoph H. Seibt, LL.M., RA FAStR, Attorney-at-Law (New York), Hamburg

Dr. Ulrich Sittard, RA, Köln

Prof. Dr. Heinrich M. Stindt, RA, Köln

Kathrin Thies, Ri'inArbG, Magdeburg

Prof. Dr. Gregor Thüsing, LL.M.,

Universitätsprofessor,

Attorney-at-Law (New York), Bonn

Prof. Dr. Kerstin Tillmanns,

Universitätsprofessorin, Hagen

Dr. Jürgen Treber, RiBAG, Erfurt

Prof. Dr. Heinz Josef Willemsen,

RA FAArbR, Düsseldorf

Werner Ziemann, VorsRiLAG, Hamm 


\section{Zitierempfehlung:}

HWK/Bearbeiter, 7. Aufl., § ... Gesetz ... Rz. ...

\section{Bibliografische Information}

der Deutschen Nationalbibliothek

Die Deutsche Nationalbibliothek verzeichnet diese

Publikation in der Deutschen Nationalbibliografie; detaillierte bibliografische Daten sind im Internet über http://dnb.d-nb.de abrufbar.

Verlag Dr. Otto Schmidt KG

Gustav-Heinemann-Ufer 58, 50968 Köln

Tel. 02 21/93738-01, Fax 02 21/93738-943

info@otto-schmidt.de

www.otto-schmidt.de

ISBN 978-3-504-42691-0

C 2016 by Verlag Dr. Otto Schmidt KG, Köln

Das Werk einschließlich aller seiner Teile ist urheberrechtlich geschützt. Jede Verwertung, die nicht ausdrücklich vom Urheberrechtsgesetz zugelassen ist, bedarf der vorherigen Zustimmung des Verlages. Das gilt insbesondere für Vervielfältigungen, Bearbeitungen, Übersetzungen, Mikroverfilmungen und die Einspeicherung und Verarbeitung in elektronischen Systemen.

Das verwendete Papier ist aus chlorfrei gebleichten Rohstoffen hergestellt, holz- und säurefrei, alterungsbeständig und umweltfreundlich.

Einbandgestaltung: Jan P. Lichtenford, Mettmann

Satz: Schäper, Bonn

Druck und Verarbeitung: Kösel, Krugzell

Printed in Germany 